\title{
Covers of Point-Hyperplane Graphs
}

\author{
ARJEH M. COHEN \\ a.m.cohen@tue.nl \\ E.J. POSTMA \\ e.j.postma@tue.nl \\ Department of Mathematics and Computer, Science, Technische Universiteit Eindhoven, Department of Mathe- \\ matics and Computer Science, Den Dolech 2, Eindhoven, The Netherlands
}

Received November 15, 2003; Revised February 9, 2005; Accepted April 25, 2005

Abstract. A cover of the non-incident point-hyperplane graph of projective dimension 3 for fields of characteristic 2 is constructed. For fields $\mathbb{F}$ of even order larger than 2, this leads to an elementary construction of the non-split extension of $\mathrm{SL}_{4}(\mathbb{F})$ by $\mathbb{F}^{6}$.

Keywords: group extension, graph cover, special linear group, projective geometry

\section{Introduction}

The non-incident point-hyperplane graph $H_{n}(\mathbb{F})$ has as vertex set the non-incident pairs of a point and a hyperplane in the projective geometry of projective dimension $n$ over a field $\mathbb{F}$. Two distinct vertices are adjacent if the points and hyperplanes are mutually incident. These graphs have been studied extensively, cf. Gramlich [4]. One of their properties is that $H_{n+1}(\mathbb{F})$ is locally $H_{n}(\mathbb{F})$ for all $\mathbb{F}$ and $n$, and that every connected and locally $H_{n}(\mathbb{F})$ graph is isomorphic to $H_{n+1}(\mathbb{F})$ whenever $n>2$.

This property does not necessarily hold if $n \leq 2$. Indeed, if $n \in\{0,1\}$ then it is easily seen not to hold. In Gramlich [4], a covering graph of $H_{3}\left(\mathbb{F}_{2}\right)$, constructed by means of a computer algebra computation, shows that it does not hold in dimension $n=2$ over $\mathbb{F}=\mathbb{F}_{2}$. In this paper we give a computer-free construction of a covering graph of $H_{3}(\mathbb{F})$ for char $\mathbb{F}=2$, thus providing counterexamples to the local recognizability of $H_{3}(\mathbb{F})$ for a wider class. This is the content of the main theorem:

Theorem 1.1 Let $q$ be a power of 2. Then there is a $q^{6}$-cover of $H_{3}\left(\mathbb{F}_{q}\right)$ which is locally $\mathrm{H}_{2}\left(\mathbb{F}_{q}\right)$ and whose automorphism group contains an extension of $\mathrm{SL}(4, q)$ by an irreducible 6-dimensional module. This extension is split only if $q=2$.

The existence part of the proof (see Theorem 3.6) is based on a construction developed in Sections 2 and 3. These sections are based on the second author's Masters' thesis [8].

In Section 5 we find automorphisms of this covering graph, generating an extension of $\mathrm{SL}_{4}(\mathbb{F})$ by $\mathbb{F}^{6}$, which is non-split if $\mathbb{F} \neq \mathbb{F}_{2}$ (see Theorem 5). Since our module is, up to a field twist, the second exterior wedge of the natural module, by the Klein correspondence we are dealing with an extension of $O^{+}(6, q)$ by its natural module. Therefore, the non-split extension is the one found by Griess [5], Sah [9], and Bell [1]. 


\subsection{Notation and conventions}

We let groups act on the right. All graphs are simple and undirected. The adjacency of vertices $u$ and $v$ is denoted by $u \perp v$. For a graph $\Gamma$, we let $V(\Gamma)$ be the set of its vertices and $D(\Gamma)$ be the set of its darts or oriented edges; that is, the set of ordered pairs of vertices $(u, v)$ for which $u \perp v$.

\section{Voltage assignments}

In this section, we discuss a general method of constructing covers of a given graph by means of voltage assignments. For a general introduction to voltage assignments, see Malnič et al. [7].

For all vertices $v$ of a graph, we call the induced graph on the neighbourhood of $v$ the local graph at $v$. Let $\Gamma$ and $\Delta$ be two connected graphs. If a map $\alpha: \Gamma \rightarrow \Delta$ preserves adjacency and if $\alpha$ maps the local graph at every vertex of $\Gamma$ isomorphically to the local graph at its image, we call $\Gamma$ a geometric cover of $\Delta$.

Let $N$ be a group. A map $\ell: D(\Delta) \rightarrow N$ such that $\ell(u, v)=\ell(v, u)^{-1}$ is called a voltage assignment of $\Delta$. We will often write $\ell_{u, v}$ for $\ell(u, v)$, or $\ell_{i, j}$ if $u=v_{i}$ and $v=v_{j}$. The lift of $\Delta$ with respect to $\ell$ is the graph with vertex set $V(\Delta) \times N$, where $(u, m) \perp(v, n)$ if and only if $u \perp v$ and $\ell(u, v)=m n^{-1}$. So $N$ acts as an automorphism group on the lift of $\Delta$ as $(v, n)^{k}=(v, n k)$, where $v \in V(\Delta)$ and $n, k \in N$.

Given a walk $P=\left(v_{0}, v_{1}, \ldots, v_{i}\right)$, where $v_{i} \perp v_{i+1}$, we call $\ell_{0,1} \ell_{1,2} \ldots \ell_{n-1, n}$ the voltage of $P$, denoted by $\ell(P)$. Using induction it is immediate that for any $m \in N$, there exists exactly one walk in the lift from $\left(v_{0}, m\right)$ to $\left(v_{n}, \ell(P)^{-1} m\right)$ that projects to $P$.

We start with two observations from Gross and Tucker [6]. Let $\Delta$ be a connected graph with voltage assignment $\ell: D(\Delta) \rightarrow N$. Let $\Gamma$ be the lift of $\Delta$ with respect to $\ell$. Then $\Gamma$ is connected if and only if for every $n \in N$ and every $v_{0} \in V(\Delta)$, there is an $i \in \mathbb{N}$ and a closed walk $\left(v_{0}, v_{1}, \ldots, v_{i}=v_{0}\right)$ such that $\ell_{0,1} \ell_{1,2} \ldots \ell_{i-1, i}=n$. The proof is easy, and so is the proof of the following lemma.

Lemma 2.1 Let $\Delta$ be a connected graph with voltage assignment $\ell: D(\Delta) \rightarrow N$. Let $\Gamma$ be the lift of $\Delta$ with respect to $\ell$. For all $n \in N$ and $v \in V(\Delta)$, the local graph at $(v, n)$ in $\Gamma$ is isomorphic to the local graph at $v$ in $\Delta$, if and only if for every triangle $u, v, w$ of $\Delta$, we have $\ell_{u, v} \ell_{v, w} \ell_{w, u}=1$.

These two observations lead to the following straightforward lemma.

Lemma 2.2 Let $\Delta$ be a connected graph with voltage assignment $\ell^{\prime}: D(\Delta) \rightarrow N^{\prime}$. Let $T$ be the normal closure of the subgroup of $N^{\prime}$ generated by the voltages of all triangles.

Let $N$ be the quotient of $N^{\prime}$ by $T$ and let $\ell_{u, v}=T \ell_{u, v}^{\prime}$. Let $M$ be the subgroup of $N$ generated by the voltages (with respect to $\ell$ ) of all closed walks. Let $\Gamma$ be the lift of $\Delta$ with respect to $\ell$.

Then by the map $(v, n) \mapsto v$, each connected component of $\Gamma$ is an $|M|$-fold geometric cover of $\Delta$. 
Let $G$ be a group of automorphisms of $\Delta$ with an action on $N$. We will say that $\ell$ is $G$-equivariant if and only if for all $g \in G$ and $v \perp w \in \Delta$, we have that $\ell_{v^{g}, w^{g}}=\left(\ell_{v, w}\right)^{g}$.

Group-equivariant voltage assignments enable the group to lift to a group of automorphisms of the lift. This is the content of the next lemma, the proof of which is again straightforward. It occurs as Proposition 19.3 in Biggs [2]. Recall that multiplication on $G \ltimes N$ is defined by $(g, k)\left(g^{\prime}, k^{\prime}\right)=\left(g g^{\prime}, k^{g^{\prime}} k\right)$.

Lemma 2.3 Let $G$ be a subgroup of Aut $\Delta$ such that $\ell$ is $G$-equivariant. Let $\Gamma$ be the lift of $\Delta$ with respect to $\ell$. Then $G \ltimes N$ acts faithfully on $\Gamma$ by the action $(v, n)^{(g, k)}=\left(v^{g}, n^{g} k\right)$.

Now suppose we have the setup of Lemma 2.2. Let $M$ be Abelian and let $\ell$ be $G$ equivariant. Choose a vertex $v \in V(\Delta)$. For all $g \in G$, choose a walk $P_{g}$ from $v^{g}$ to $v$, and let $\lambda(g)$ be the voltage of $P_{g}$. Choose $P_{1}$ such that $\lambda(1)=0$. Then the following lemma holds.

Lemma 2.4 The stabilizer in $G \ltimes N$ of the connected component $\Gamma_{0}$ of $\Gamma$ containing $(v, 0)$ is $H=\{(g, \lambda(g)+m) \mid g \in G, m \in M\}$, which is an extension of $G$ by $M$.

Proof: Since $(v, 0)^{(g, \lambda(g)+m)}=\left(v^{g}, \lambda(g)+m\right)$ and since the walk in $\Gamma$ starting at $\left(v^{g}, \lambda(g)+\right.$ $m$ ) and projecting down to $P_{g}$ ends at $(v, m)$, we have that $H$ stabilizes $\Gamma_{0}$. Conversely, if an element $(g, n)$ stabilizes $\Gamma_{0}$, it maps $(v, 0)$ to an element $\left(v^{g}, n\right)$ such that there is a walk from $\left(v^{g}, \lambda(g)\right)$ to $\left(v^{g}, n\right)$. Then the projection of that walk down to $\Delta$ is a closed walk; hence $\lambda(g)-n \in M$. So $H$ is the full stabilizer of $\Gamma_{0}$.

The kernel of the projection onto the first coordinate is $\{1\} \times M$, so that is a normal subgroup. The quotient by that subgroup is $G$.

\section{3. $\mathrm{SL}(V)$-modules}

We recall some multilinear algebra in order to be able to construct the voltage assignment in the next section.

Consider the projective geometry $\mathbb{P}_{n}(\mathbb{F})$ of (projective) dimension $n$ over the field $\mathbb{F}$. We denote incidence by $\subset$ and the projective dimension by dim. Furthermore, $V$ will be the vector space $\mathbb{F}^{4}$ with basis $e_{1}, \ldots, e_{4}$ and dual basis $f_{1}, \ldots, f_{4}$, so $\mathbb{P}_{3}(\mathbb{F})=\mathbb{P}(V)$. Let

$$
\left.\bigwedge^{k} V=V^{\otimes k} /\left\langle v_{1} \otimes v_{2} \otimes \cdots \otimes v_{k}\right| v_{i}=v_{j} \text { for some } i \neq j\right\rangle
$$

be the $k$ th Grassmannian of $V$. The image of $v_{1} \otimes \cdots \otimes v_{k}$ in $\bigwedge^{k} V$ is denoted $v_{1} \wedge \cdots \wedge v_{k}$. Let $G$ be a group with a linear action on $V$; this induces a natural action on $\wedge^{k} V$. Now $G \leq \operatorname{SL}(V)$ if and only if $G$ stabilizes every element of $\wedge^{4} V$. We will mostly be using the case where $k=2$. We need the following elementary lemmas.

Lemma 3.1 There is a canonical isomorphism from $\left(\bigwedge^{2} V\right)^{*}$ to $\bigwedge^{2}\left(V^{*}\right)$ that preserves the induced action of $\mathrm{GL}(V)$. 
Sketch of Proof: Let $B^{*}: \bigwedge^{2}\left(V^{*}\right) \times \bigwedge^{2} V \rightarrow \mathbb{F}$ be defined for $\hat{h}=h_{1} \wedge h_{2} \in \bigwedge^{2}\left(V^{*}\right)$ and $\hat{v}=v_{1} \wedge v_{2} \in \bigwedge^{2} V$ by

$$
B^{*}(\hat{h}, \hat{v})=h_{1}\left(v_{1}\right) h_{2}\left(v_{2}\right)-h_{1}\left(v_{2}\right) h_{2}\left(v_{1}\right),
$$

and extended bilinearly. As $B^{*}$ is nondegenerate, $\hat{h} \mapsto\left(\hat{v} \mapsto B^{*}(\hat{h}, \hat{v})\right)$ is an isomorphism $\left(\bigwedge^{2} V\right)^{*} \rightarrow \bigwedge^{2}\left(V^{*}\right)$ respecting the induced action of $\operatorname{GL}(V)$.

Because of the preceding lemma we can drop the parentheses in the future and write $\bigwedge^{2} V^{*}$. Fix an isomorphism $\chi: \bigwedge^{4} V \rightarrow \mathbb{F}$.

Lemma 3.2 Let $V$ be a vector space of dimension 4 over a field $\mathbb{F}$. Then there is a canonical isomorphism $\psi: \bigwedge^{2} V \rightarrow \bigwedge^{2} V^{*}$ with inverse $\phi$, which respects the natural induced group actions of $\mathrm{SL}_{4}(\mathbb{F})$ on $\bigwedge^{2} V$ and $\bigwedge^{2} V^{*}$.

Sketch of Proof: We define $B: \bigwedge^{2} V \times \bigwedge^{2} V \rightarrow \mathbb{F}$ as follows:

$$
B\left(v_{1} \wedge v_{2}, w_{1} \wedge w_{2}\right)=\left(v_{1} \wedge v_{2} \wedge w_{1} \wedge w_{2}\right)^{\chi},
$$

and extended by linearity. Then $B$ is nondegenerate. Now let $\psi$ map $\hat{w} \in \bigwedge^{2} V$ to the linear functional that maps $\hat{v} \in \bigwedge^{2} V$ to $B(\hat{v}, \hat{w})$. Then $\psi$ is a vector space isomorphism.

Whenever we consider it appropriate, we will omit $\psi$ and $\phi$.

Lemma 3.3 Let $h_{1}, h_{2}$ be linearly independent elements of $V^{*}$ and let $v_{1}, v_{2}$ be linearly independent elements of $V$ such that $h_{i}\left(v_{j}\right)=0$. Then $\left(v_{1} \wedge v_{2}\right)^{\psi}=\alpha h_{1} \wedge h_{2}$ for some $\alpha \in \mathbb{F}$.

Proof: $\quad$ Let $K=\operatorname{Ker} h_{1} \cap \operatorname{Ker} h_{2}=\left\langle v_{1}, v_{2}\right\rangle$. Put $\hat{v}=\left(v_{1} \wedge v_{2}\right)^{\psi}$ and $\hat{h}=h_{1} \wedge h_{2}$.

Let $w_{1}, w_{2} \in V$ and write $\hat{w}=w_{1} \wedge w_{2}$. We will first show that $\hat{h}(\hat{w})=0$ precisely if $\hat{v}(\hat{w})=0$. We may assume $\hat{w} \neq 0$. We can move $w_{1}$ to any projective point on the projective line $\left\langle w_{1}, w_{2}\right\rangle$ keeping the same value for $\hat{w}$ by either switching $w_{1}$ and $w_{2}$ or replacing $w_{1}$ by $w_{1}+r w_{2}$ for some field element $r$. So if $\left\langle w_{1}, w_{2}\right\rangle$ intersects $K$, then we may assume that the point of intersection is $w_{1}$. Then

$$
\begin{aligned}
& \hat{v}(\hat{w})=v_{1} \wedge v_{2} \wedge w_{1} \wedge w_{2}=0, \\
& \hat{h}(\hat{w})=h_{1}\left(w_{1}\right) h_{2}\left(w_{2}\right)-h_{1}\left(w_{2}\right) h_{2}\left(w_{1}\right)=0 .
\end{aligned}
$$

Otherwise, $\left\langle v_{1}, v_{2}, w_{1}, w_{2}\right\rangle=V$ so $\hat{v}(\hat{w}) \neq 0$. So $\hat{v}(\hat{w})=0$ precisely if $\left\langle w_{1}, w_{2}\right\rangle$ intersects $K$, and in that case we also have $\hat{h}(\hat{w})=0$.

Now suppose $\hat{h}(\hat{w})=0$. Then $h_{1}\left(w_{1}\right) h_{2}\left(w_{2}\right)=h_{1}\left(w_{2}\right) h_{2}\left(w_{1}\right)$. Let $w=f_{1}\left(w_{2}\right) w_{1}-$ $f_{1}\left(w_{1}\right) w_{2}$. Then

$$
\begin{aligned}
& f_{1}(w)=f_{1}\left(w_{2}\right) f_{1}\left(w_{1}\right)-f_{1}\left(w_{1}\right) f_{1}\left(w_{2}\right)=0, \\
& f_{2}(w)=f_{1}\left(w_{2}\right) f_{2}\left(w_{1}\right)-f_{1}\left(w_{1}\right) f_{2}\left(w_{2}\right)=0 .
\end{aligned}
$$

So again $\hat{h}(\hat{w})=0$ precisely if $\left\langle w_{1}, w_{2}\right\rangle$ intersects $K$. 
Now let $x_{1}, x_{2} \in V$ with $\hat{x}=x_{1} \wedge x_{2}$. We will show that $\hat{v}(\hat{w}) \hat{h}(\hat{x})=\hat{v}(\hat{x}) \hat{h}(\hat{w})$. We may assume that $\hat{h}$ and $\hat{v}$ are nonzero on both $\hat{w}$ and $\hat{x}$. So $\left\langle w_{1}, w_{2}\right\rangle$ intersects $\operatorname{Ker} h_{1}$ and Kerh $h_{2}$ in distinct projective points. We may assume that these intersection points are $\left\langle w_{1}\right\rangle$ and $\left\langle w_{2}\right\rangle$, respectively, so $h_{i}\left(w_{i}\right)=0$. Similarly we may assume $h_{i}\left(x_{i}\right)=0$.

Now $\left\langle v_{1}\right\rangle,\left\langle v_{2}\right\rangle,\left\langle w_{i}\right\rangle$ and $\left\langle x_{i}\right\rangle$ are four projective points in the hyperplane $\operatorname{Ker} h_{i}$, so we write $w_{i}=\alpha_{i, 1} v_{1}+\alpha_{i, 2} v_{2}+\alpha_{i, 3} x_{i}$. Then

$$
\hat{v}(\hat{w}) \hat{h}(\hat{x})=-\alpha_{1,3} \alpha_{2,3}\left(v_{1} \wedge v_{2} \wedge x_{1} \wedge x_{2}\right)\left(h_{1}\left(x_{2}\right) h_{2}\left(x_{1}\right)\right)
$$

and

$$
\hat{v}(\hat{x}) \hat{h}(\hat{w})=-\alpha_{1,3} \alpha_{2,3}\left(v_{1} \wedge v_{2} \wedge x_{1} \wedge x_{2}\right)\left(h_{1}\left(x_{2}\right) h_{2}\left(x_{1}\right)\right) .
$$

It follows that $\hat{v}$ and $\hat{h}$ differ by the same factor on all elements of shape $w_{1} \wedge w_{2}$, and therefore on all of $\bigwedge^{2} V$.

For an arbitrary vector space $Y$, we let

$$
S_{2}(Y)=(Y \otimes Y) /\langle v \otimes w-w \otimes v \mid v, w \in Y\rangle
$$

be the second order symmetric tensor of $Y$. Then the natural action of $\operatorname{SL}(Y)$ on $Y \otimes Y$ induces a natural action on $S_{2}(Y)$. We denote the image of $v \otimes w$ in $S_{2}(Y)$ by $v w$. We will often write $w^{2}$ for $w w$.

Now let char $\mathbb{F}=2$, and let $W=\bigwedge^{2} V=\bigwedge^{2}\left(V^{*}\right)$ of dimension 6. Then $S_{2}(W)$ has dimension 21. The subspace $W^{(2)}$ of $S_{2}(W)$, defined as

$$
W^{(2)}=\left\langle\hat{w}^{2} \mid \hat{w} \in W\right\rangle,
$$

has dimension 6 and is invariant under the induced action of $\mathrm{GL}(V)$.

Lemma 3.4 Let $w, x, y, z \in V$ be such that $w \wedge x \wedge y \wedge z=1$. Then the vector

$$
U=(w \wedge x)(y \wedge z)+(w \wedge y)(z \wedge x)+(w \wedge z)(x \wedge y)
$$

does not depend on the choice of $w, x, y, z$ and is fixed by $\mathrm{SL}(V)$.

Proof: The map

$$
\Delta:(w, x, y, z) \mapsto(w \wedge x)(y \wedge z)+(w \wedge y)(z \wedge x)+(w \wedge z)(x \wedge y)
$$

is 4-linear and alternating. There is only one such map, up to scalar multiples: the determinant. Hence for tuples of vectors such that $\operatorname{det}(w, x, y, z)=w \wedge x \wedge y \wedge z=1$, we find that $\Delta$ must be constant.

Since the image of $\Delta(w, x, y, z)$ under an element of $\operatorname{SL}(V)$ is $\Delta\left(w^{\prime}, x^{\prime}, y^{\prime}, z^{\prime}\right)$ for some tuple satisfying $w^{\prime} \wedge x^{\prime} \wedge y^{\prime} \wedge z^{\prime}=1$, the element $U$ is fixed by $\operatorname{SL}(V)$. 


\section{The graph and its voltage assignment}

Following Gramlich [4], we define the graph $H_{3}(\mathbb{F})$ to have vertex set

$$
\left\{(x, X) \mid x, X \in \mathbb{P}_{3}(\mathbb{F}), \operatorname{dim} x=0, \operatorname{dim} X=2, x \not \subset X\right\}
$$

and adjacency defined by

$$
(x, X) \perp(y, Y) \Leftrightarrow x \subset Y \text { and } y \subset X .
$$

We require char $\mathbb{F}=2$ and we retain $V, W, W^{(2)}$ and $U$ as in the previous section.

The computer algebra computations of Gramlich [4] indicated that it might be possible to find a 6-dimensional module to extend $\mathrm{SL}_{4}\left(\mathbb{F}_{2}\right)$ with and obtain the automorphism group of the cover of $H_{3}\left(\mathbb{F}_{2}\right)$. It seemed natural that this module would be $\bigwedge^{2} V$. On the other hand, the vertices of the graph could be modelled as projective points in $V \otimes V^{*}$ with edges corresponding to projective points in $S_{2}\left(V \otimes V^{*}\right)$. By the composition of natural maps

$$
S_{2}\left(V \otimes V^{*}\right) \rightarrow \bigwedge^{2} V \otimes \bigwedge^{2} V^{*}=\bigwedge^{2} V \otimes \bigwedge^{2} V \rightarrow S_{2}\left(\bigwedge^{2} V\right)
$$

we could map an edge into an $\mathrm{SL}_{4}(\mathbb{F})$-module containing a twisted copy of $\bigwedge^{2} V$, viz. $W^{(2)}$. The composition of these maps gives the setting for our voltage assignment.

We will often represent a vertex $(x, X)$ of $H_{3}(\mathbb{F})$ by a pair $(v, h)$ of a nonzero vector $v$ in $x$ and a functional $h$ with kernel $X$. Let $\left(v_{1}, h_{1}\right) \perp\left(v_{2}, h_{2}\right)$ be two adjacent vertices in $H_{3}(\mathbb{F})$. We let $\ell: D\left(H_{3}(\mathbb{F})\right) \rightarrow S_{2}(W)$ assign the voltage

$$
h_{1}\left(v_{1}\right)^{-1} h_{2}\left(v_{2}\right)^{-1}\left(v_{1} \wedge v_{2}\right)\left(h_{1} \wedge h_{2}\right)^{\phi}
$$

to the dart from $\left(v_{1}, h_{1}\right)$ to $\left(v_{2}, h_{2}\right)$. Note that this is independent of the representatives $v_{i}$ and $h_{i}$. We will often choose $v$ and $h$ such that $h(v)=1$.

We will sometimes regard $S_{2}(W)$ as a group only, so the subgroups are the subspaces over $\mathbb{F}_{2}$-not necessarily over $\mathbb{F}$. We denote the $\mathbb{F}_{2}$-linear span of $v_{0}, \ldots, v_{k}$ by $\left\langle v_{0}, \ldots, v_{k}\right\rangle_{\mathbb{F}_{2}}$. Let $\ell^{U}: D\left(H_{3}(\mathbb{F})\right) \rightarrow S_{2}(W) /\langle U\rangle_{\mathbb{F}_{2}}$ be the composition of $\ell$ with the natural projection of $S_{2}(W)$ to $S_{2}(W) /\langle U\rangle_{\mathbb{F}_{2}}$. Note that both $\ell$ and $\ell^{U}$ are $\mathrm{SL}_{4}(\mathbb{F})$-equivariant.

The following theorem shows the existence of a $q^{6}$-fold geometric cover of $H_{3}\left(\mathbb{F}_{q}\right)$ if $q$ is even, and so provides infinitely many counterexamples to the extension of Theorem 1.3.21 of Gramlich [4] for $H_{n+1}\left(\mathbb{F}_{q}\right)$ to $n=2$. This proves the existence part of Theorem 1.1.

Theorem 4.1 Let char $\mathbb{F}=2$. Let $\Gamma$ be the lift of $H_{3}(\mathbb{F})$ with respect to $\ell^{U}$. Then every connected component of $\Gamma$ is an $\left|\mathbb{F}^{6}\right|$-fold geometric cover of $H_{3}(\mathbb{F})$.

For proving Theorem 4.1. we need some auxiliary lemmas which are of interest in their own right, since they provide information on the geometric covers of $H_{3}(\mathbb{F})$ in general. We will use the words triangle, quadrangle and pentagon to mean closed walks of the obvious lengths consisting of different vertices. A quadrangle will be called special of type $A$ if the number of distinct (projective) points, or the number of distinct hyperplanes, occurring in 
its vertices, is two; it will be called special of type $B$ if the number of distinct points and the number of distinct hyperplanes occurring are both three.

Consider the following chain complex over $\mathbb{F}_{2}$. We let $C_{0}$ and $C_{1}$ be the free modules spanned by the vertices and edges of $H_{3}(\mathbb{F})$, respectively. The boundary map $\partial_{1}: C_{1} \rightarrow C_{0}$ maps an edge to the sum of its vertices. We let $C_{2}$ be the trivial module and therefore $\partial_{2}$ is the zero map. In this manner we have an explicit description of the homology group $H_{1}=H_{1}\left(C_{*}, \mathbb{F}_{2}\right)=\operatorname{ker} \partial_{1}$. Let $Q$ be the submodule of $H_{1}$ spanned by triangles and special quadrangles of type $A$. In Proposition 4.4 we will show that each closed walk is an element of $Q$; that is, $Q=H_{1}$.

Lemma 4.2 The sum of edges of a quadrangle is in $Q$.

Proof: Consider the quadrangle $\left(v_{0}, h_{0}\right), \ldots,\left(v_{3}, h_{3}\right)$. We may assume that these representatives have been chosen such that $h_{i}\left(v_{i}\right)=1$. Suppose that for some $i$, we have $h_{i}\left(v_{i+2}\right)=h_{i+2}\left(v_{i}\right)=0$; say for $i=0$. Then the quadrangle consists of two triangles as depicted in figure 1 (a).

Now consider a quadrangle where for all $i$ either $h_{i}\left(v_{i+2}\right)$ or $h_{i+2}\left(v_{i}\right)$ is nonzero; we may assume that $h_{0}\left(v_{2}\right)=h_{1}\left(v_{3}\right)=1$. Then we can split the quadrangle into four special quadrangles by adding the vertices $\left(v_{2}, h_{0}\right)$ and $\left(v_{3}, h_{1}\right)$, as depicted in figure 1(b) and specified in the table below.

\begin{tabular}{cccc|r}
\multicolumn{4}{c|}{ Vertices } & Type \\
\hline$\left(v_{0}, h_{0}\right)$ & $\left(v_{1}, h_{1}\right)$ & $\left(v_{2}, h_{0}\right)$ & $\left(v_{3}, h_{1}\right)$ & $A$ \\
$\left(v_{1}, h_{1}\right)$ & $\left(v_{2}, h_{2}\right)$ & $\left(v_{3}, h_{1}\right)$ & $\left(v_{2}, h_{0}\right)$ & $B$ \\
$\left(v_{2}, h_{2}\right)$ & $\left(v_{3}, h_{3}\right)$ & $\left(v_{2}, h_{0}\right)$ & $\left(v_{3}, h_{1}\right)$ & $A$ \\
$\left(v_{3}, h_{3}\right)$ & $\left(v_{0}, h_{0}\right)$ & $\left(v_{3}, h_{1}\right)$ & $\left(v_{2}, h_{0}\right)$ & $B$
\end{tabular}

If the added vertices coincide with vertices of the quadrangle, then instead the quadrangle is split into two special quadrangles or the quadrangle itself is special.

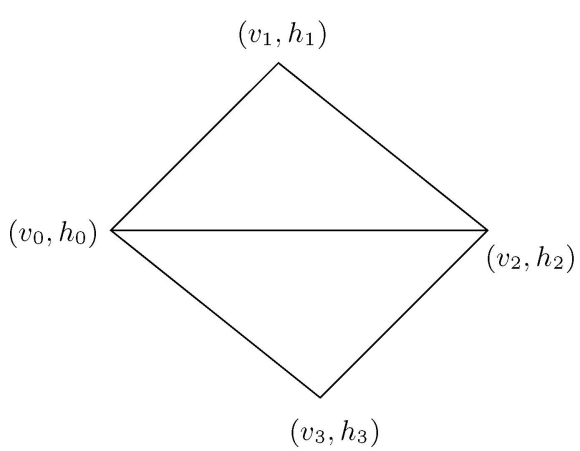

(a)

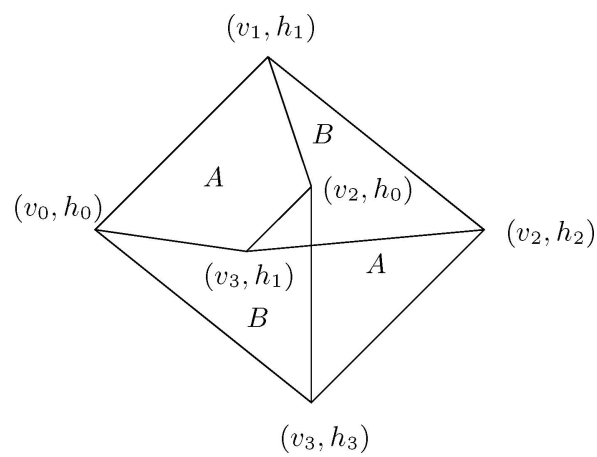

(b)

Figure 1. (a) A quadrangle consisting of two triangles. (b) Splitting a quadrangle into four special quadrangles. 


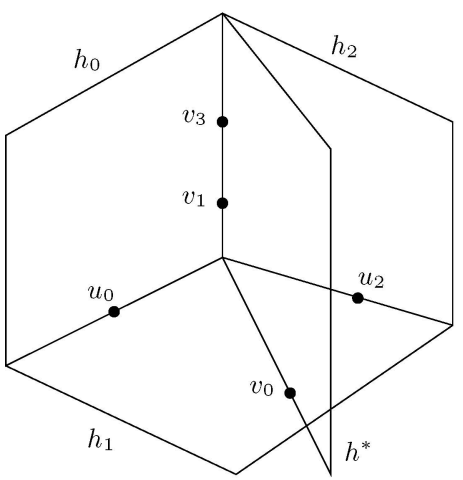

(a)

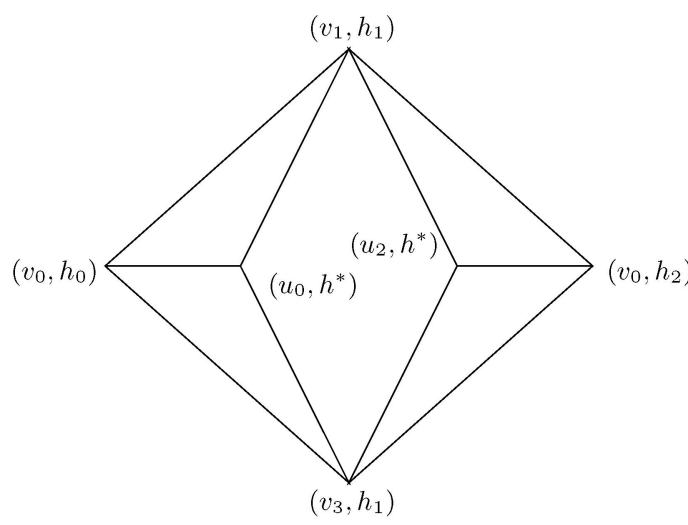

(b)

Figure 2. (a) Finding the vertices $\left(u_{0}, h^{*}\right)$ and $\left(u_{2}, h^{*}\right)$. (b) A quadrangle of type $B$ is a quadrangle of type $A$ plus four triangles.

So let us consider a special quadrangle of type $B$. It may be assumed to have vertices $\left(v_{0}, h_{0}\right),\left(v_{1}, h_{1}\right),\left(v_{0}, h_{2}\right),\left(v_{3}, h_{1}\right)$. The projective geometric relations between $v_{i}$ and $h_{j}$ are depicted in figure 2(a). Let $h^{*}$ be the hyperplane containing $v_{0}, v_{1}$ and $v_{3}$, and pick two points $u_{0}$ and $u_{2}$ such that $u_{i}$ is in the intersection of $h_{i}$ and $h_{1}$, but not in $h^{*}$. Then $\left(u_{i}, h^{*}\right)$ are vertices of $H_{3}(\mathbb{F})$, and their neighbours are as depicted in figure 2(b). We see that a quadrangle of type $B$ is the sum of a quadrangle of type $A$ and four triangles.

Lemma 4.3 The sum of edges of a pentagon is in $Q$.

Proof: Let us take a pentagon $\left(v_{0}, h_{0}\right), \ldots,\left(v_{4}, h_{4}\right)$. We choose the representatives such that $h_{i}\left(v_{i}\right)=1$ for all $i$.

Choose an index $i$ and consider the index set $\{i-2, i, i+2\}$ (modulo 5). Now suppose that the common null space $\mathcal{N}_{i}$ of $h_{i-2}, h_{i}$ and $h_{i+2}$ is not contained in $\mathcal{V}_{i}=\left\langle v_{i-2}, v_{i}, v_{i+2}\right\rangle$. Then take some $v \in \mathcal{N}_{i} \backslash \mathcal{V}_{i}$, and some $h \in V^{*}$ such that $h$ is zero on $\mathcal{V}_{i}$, but not on $v$. Then the vertex $(v, h)$ is adjacent to $\left(v_{i-2}, h_{i-2}\right),\left(v_{i}, h_{i}\right)$ and $\left(v_{i+2}, h_{i+2}\right)$. Hence the pentagon is the sum of two quadrangles and a triangle, as in figure 3 .

Now suppose that for all indices $i$, we have that $\mathcal{N}_{i} \subseteq \mathcal{V}_{i}$. Since $\mathcal{N}_{i}$ has a positive vector space dimension, there is a nonzero vector $v$ in $\mathcal{V}_{i}$ on which $h_{i-2}, h_{i}$ and $h_{i+2}$ are all zero. Let

$$
v=\lambda_{i-2} v_{i-2}+\lambda_{i} v_{i}+\lambda_{i+2} v_{i+2} .
$$

If $\lambda_{i}=0$, then also

$$
0=h_{i \pm 2}\left(\lambda_{i \pm 2} v_{i \pm 2}+\lambda_{i \mp 2} v_{i \mp 2}\right)=\lambda_{i \pm 2},
$$




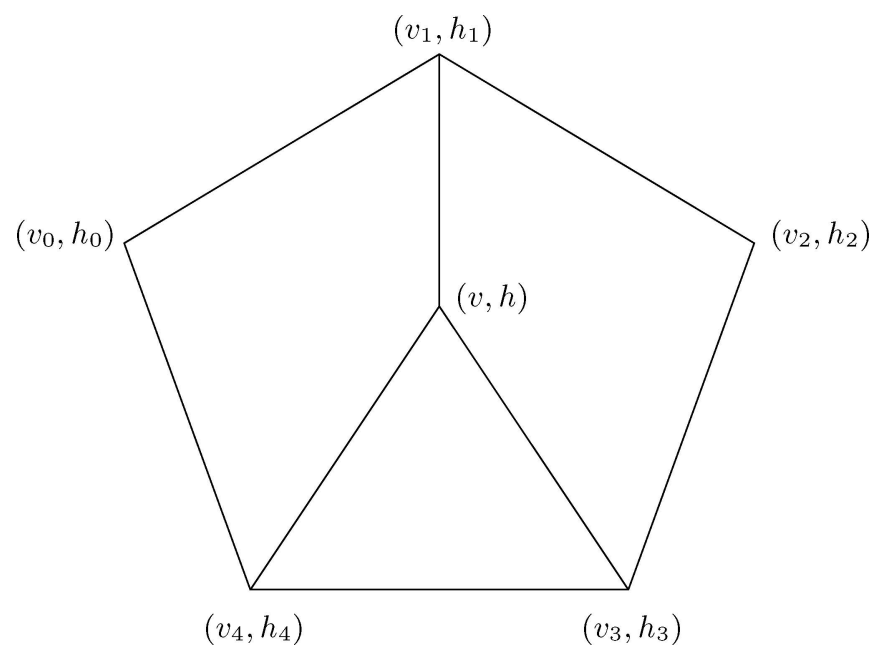

Figure 3. Splitting a pentagon into a triangle and two quadrangles.

contradicting $v \neq 0$. So we may assume $\lambda_{i}=1$. Then

$$
\begin{aligned}
& 0=h_{i}(v)=\lambda_{i-2} h_{i}\left(v_{i-2}\right)+1+\lambda_{i+2} h_{i}\left(v_{i+2}\right) ; \\
& 0=h_{i-2}(v)=\lambda_{i-2}+h_{i-2}\left(v_{i}\right) \\
& 0=h_{i+2}(v)=\lambda_{i+2}+h_{i+2}\left(v_{i}\right) .
\end{aligned}
$$

So we find $\lambda_{i \pm 2}=h_{i \pm 2}\left(v_{i}\right)$ and hence

$$
h_{i-2}\left(v_{i}\right) h_{i}\left(v_{i-2}\right)+h_{i+2}\left(v_{i}\right) h_{i}\left(v_{i+2}\right)=1 .
$$

If we sum Eq. (2) over all $i$ the right hand side is 1 . But every term on the left hand side occurs twice, so the left hand side is 0 . Contradiction.

Proposition 4.4 $Q=H_{1}$.

Proof: It is sufficient to show that the sum of edges of any closed walk is in $Q$. Lemmas 4.2 and 4.3 tell us that the statement holds for all closed walks of length at most 5. Let $c=$ $\left(v_{0}, v_{1}, \ldots, v_{n}=v_{0}\right)$ be a shortest closed walk not in $Q$; so $n \geq 6$. By Lemma 1.3.5 of Gramlich [4], the diameter of $H_{3}(\mathbb{F})$ is two, so there is a path of length at most 2 from $v_{0}$ to $v_{3}$. Let us call this path $P$. This gives us two new closed walks: the first one is formed by $v_{0}, \ldots, v_{3}$, followed by the reverse of $P$ - the length of which is at most 5 , whence it is in $Q$; the second one is formed by $P$, followed by $v_{4}, \ldots, v_{n}$ - the length of which is at most $n-1$, whence it is also in $Q$. Therefore $c$ is also in $Q$. Contradiction.

Note that this proposition can also be shown to hold for homology over $\mathbb{Z}$. 
Lemma 4.5 The voltage of a triangle with respect to $\ell$ is $U$.

Proof: Let $\left(v_{1}, h_{1}\right),\left(v_{2}, h_{2}\right),\left(v_{3}, h_{3}\right)$ be a triangle in $H_{3}(\mathbb{F})$. We assume $h_{i}\left(v_{i}\right)=1$. Then $\left\{v_{i}\right\}$ and $\left\{h_{i}\right\}$ are both linearly independent sets. Hence the intersection of the null spaces of $\left\{h_{i}\right\}$ has dimension 1 ; choose $u$ nonzero in it. Then $\left\{v_{1}, v_{2}, v_{3}, u\right\}$ form a basis for $V$. We may assume that $v_{1} \wedge v_{2} \wedge v_{3} \wedge u=1$.

Since $h_{1}$ and $h_{2}$ both vanish on $v_{3}$ and $u$, we have $\alpha\left(h_{1} \wedge h_{2}\right)^{\phi}=v_{3} \wedge u$ for some nonzero $\alpha \in \mathbb{F}$ by Lemma 3.3. Now $1=v_{1} \wedge v_{2} \wedge v_{3} \wedge u=\alpha\left(h_{1}\left(v_{1}\right) h_{2}\left(v_{2}\right)+h_{1}\left(v_{2}\right) h_{2}\left(v_{1}\right)\right)=\alpha$. Hence $\left(h_{1} \wedge h_{2}\right)^{\phi}=v_{3} \wedge u$; similarly we obtain $\left(h_{1} \wedge h_{3}\right)^{\phi}=v_{2} \wedge u$ and $\left(h_{2} \wedge h_{3}\right)^{\phi}=v_{1} \wedge u$. So if $\{i, j, k\}=\{1,2,3\}$, then the voltage of the dart from $\left(v_{i}, h_{i}\right)$ to $\left(v_{j}, h_{j}\right)$ is $\left(v_{i} \wedge v_{j}\right)$ $\left(v_{k} \wedge u\right)$. The sum of the voltages is then

$$
\left(v_{1} \wedge v_{2}\right)\left(v_{3} \wedge u\right)+\left(v_{1} \wedge v_{3}\right)\left(v_{2} \wedge u\right)+\left(v_{2} \wedge v_{3}\right)\left(v_{1} \wedge u\right)=U
$$

Lemma 4.6 The voltage of a closed walk in $H_{3}(\mathbb{F})$ with respect to $\ell$ is in $W^{(2)} \oplus\langle U\rangle_{\mathbb{F}_{2}}$.

Proof: By Proposition 4.4, the voltage of any closed walk can be written as the sum of voltages of triangles and special quadrangles of type $A$. By Lemma 4.5 , the voltage of a triangle is $U$.

Now consider a special quadrangle of type $A$. We may, by duality, assume its vertices are $\left(v_{0}, h_{0}\right),\left(v_{1}, h_{1}\right),\left(v_{0}, h_{2}\right)$ and $\left(v_{1}, h_{3}\right)$ with $h_{i}\left(v_{i}\right)=h_{i+2}\left(v_{i}\right)=1$. By Lemma 3.3 , its voltage is

$$
\begin{aligned}
& \left(v_{0} \wedge v_{1}\right)\left(h_{0} \wedge h_{1}+h_{1} \wedge h_{2}+h_{2} \wedge h_{3}+h_{3} \wedge h_{0}\right)^{\phi} \\
& \quad=\left(v_{0} \wedge v_{1}\right)\left(\left(h_{0}+h_{2}\right) \wedge\left(h_{1}+h_{3}\right)\right)^{\phi}=\alpha\left(v_{0} \wedge v_{1}\right)^{2} \in W^{(2)}
\end{aligned}
$$

where $\alpha$ is some field element.

Lemma 4.7 For all $w \in W^{(2)}$ there is a closed walk in $H_{3}(\mathbb{F})$ with voltage $w$ with respect to $\ell$.

Proof: It is sufficient to show that a set of generators of $W^{(2)}$ occurs as voltages of closed walks. Note that an $\mathbb{F}$-basis is not necessarily sufficient-we need an $\mathbb{F}_{2}$-basis.

Consider the special quadrangle of type $A$ with vertices $\left(e_{3}, f_{3}\right),\left(e_{4}, f_{4}\right),\left(e_{3}, \lambda f_{2}+f_{3}\right)$ and $\left(e_{4}, f_{1}+f_{4}\right)$. Its voltage is $\lambda e_{1,2}^{2}$. By permuting the base vectors and by choosing different values for $\lambda$, we obtain an $\mathbb{F}_{2}$-basis for $W^{(2)}$.

Proof of Theorem 4.1: We apply Lemma 2.2. Lemma 4.5 gives us $T=\langle U\rangle_{\mathbb{F}_{2}}$; then Lemmas 4.6 and 4.7 imply that $M=W^{(2)}$. Hence every connected component of $\Gamma$ is a $\left|W^{(2)}\right|$-fold geometric cover of $\Delta$. Since $W^{(2)} \cong \mathbb{F}^{6}$, we have finished the proof.

Notice that $\Gamma$ is a cover of $H_{3}(\mathbb{F})$ in the sense of 2-dimensional simplicial complexes whose 2 -simplices are the triangles of the graphs. Since closed walks in $\Gamma$ correspond to closed walks in $H_{3}(\mathbb{F})$ with voltage 0 , the cover is simply connected if and only if every 
closed walk of voltage 0 in $H_{3}(\mathbb{F})$ is a sum of triangles. We conjecture that $\Gamma$ is not simply connected in this sense. For $\mathbb{F}=\mathbb{F}_{2}$ this is true because a computer computation shows that the walk with the vertices in the list below and with voltage 0 is not a sum of triangles in that case:

$$
\left(e_{1}, f_{1}\right), \quad\left(e_{2}, f_{2}+f_{4}\right), \quad\left(e_{1}, f_{1}+f_{3}\right), \quad\left(e_{2}, f_{2}\right), \quad\left(e_{1}+e_{3}, f_{1}\right), \quad\left(e_{2}+e_{4}, f_{2}\right),
$$

where $\left\{e_{i}\right\}$ and $\left\{f_{i}\right\}$ are bases of $V$ and $V^{*}$, respectively.

\section{A group of automorphisms}

Let $\Gamma$ be the graph of Theorem 4.1, so char $\mathbb{F}=2$. Set $N=S_{2}(W) /\langle U\rangle_{\mathbb{F}_{2}}$ and $M=$ $\left(W^{(2)}+\langle U\rangle_{\mathbb{F}_{2}}\right) /\langle U\rangle_{\mathbb{F}_{2}}$. When writing elements of $N$ and $M$, we will often omit the added $\langle U\rangle_{\mathbb{F}_{2}}$. The group $\mathrm{SL}_{4}(\mathbb{F})$ acts on $H_{3}(\mathbb{F})$ as follows. A group element $g$ maps a vertex $(v, h)$ to $\left(v^{g}, h^{g}\right)$, where $h^{g}(w)=h\left(w^{g^{-1}}\right)$. According to Lemma 2.3, the group $\mathrm{SL}_{4}(\mathbb{F}) \ltimes N$ acts on $\Gamma$. By Lemma 2.4 and the results of Section 4, an extension $E$ of $\mathrm{SL}_{4}(\mathbb{F})$ by $M$ acts on a connected component of $\Gamma$. The content of Theorem 5.1. below is that this extension is nonsplit unless $|\mathbb{F}|=2$. The existence of this nonsplit extension was known by Bell [1], Griess [5], and Sah [9]. The theorem proves the automorphism group part of Theorem 1.1.

An extension of a group by an Abelian group corresponds to a 2-cocycle in the standard chain complex of the group that is being extended. The extension is nonsplit exactly if the cocycle is not a 2-coboundary, see Brown [3]. In this section we find an explicit cocycle that defines this extension.

We let $i$ and $\pi$ denote the natural maps in the following diagram:

By Brown [3], a 2-cocycle is determined by a section $s: \mathrm{SL}_{4}(\mathbb{F}) \rightarrow E$ of $\pi$. It is a map $f: G \times G \rightarrow M$ such that

$$
s(g) s(h)=s(g h) i(f(g, h)), \quad f(g, 1)=f(1, g)=0 .
$$

This is the right-action version of (3.3.3) of [3]. The group law on the set $\mathrm{SL}_{4}(\mathbb{F}) \times M$ that makes it into a group isomorphic with $E$ is

$$
\left[g_{1}, m_{1}\right]\left[g_{2}, m_{2}\right]=\left[g_{1} g_{2}, m_{1}^{g_{1}}+m_{2}+f\left(g_{1}, g_{2}\right)\right] .
$$

So in order to describe the cocycle, we need to define the section $s$. The elements of $E$ are most easily described as elements of $\mathrm{SL}_{4}(\mathbb{F}) \ltimes N$. Therefore we construct a map $\lambda: \mathrm{SL}_{4}(\mathbb{F}) \rightarrow N$ such that $s(g)=(g, \lambda(g)) \in E$ and $\lambda(1)=0$. Then $f$ is determined by Eq. (3) as

$$
f(g, h)=-\lambda(g h)+\lambda(g)^{h}+\lambda(h) .
$$

We can choose $\lambda$ as in Lemma 2.4. The construction of $\lambda$ is then coordinate-dependent. In order to compute it, choose a basis $\left\{e_{i}\right\}$ for $\mathbb{F}^{4}$ and a dual basis $\left\{f_{i}\right\}$. We need to fix a 
vertex of $H_{3}(\mathbb{F})$, say $\left(e_{1}, f_{1}\right)$, and then for each $g \in \mathrm{SL}_{4}(\mathbb{F})$ we need to choose a walk from $\left(e_{1}^{g}, f_{1}^{g}\right)$ to $\left(e_{1}, f_{1}\right)$. The voltage along this walk is then $\lambda(g)$.

Theorem 5.1 Let char $\mathbb{F}=2$ and $|\mathbb{F}|>2$. Then the stabilizer $E$ in $\mathrm{SL}_{4}(\mathbb{F}) \ltimes N$ of a connected component is a non-split extension of $\mathrm{SL}_{4}(\mathbb{F})$ by $\mathbb{F}^{6}$.

Proof: Let $\alpha \in \mathbb{F}$ be an element outside the ground field. Let $F$ denote the additive subgroup $\langle 1, \alpha\rangle_{\mathbb{F}_{2}}$ of $\mathbb{F}$ of order 4 . For $x \in F$, let $A_{x}$ be the element of $\left.\mathrm{SL}_{4}(\mathbb{F})\right)$ fixing $e_{2}$ and $e_{4}$, and mapping $e_{1}$ and $e_{3}$ to $e_{1}+x e_{2}$ and $e_{3}+x e_{4}$, respectively. We will show that the subgroup $A=\left\{A_{x} \mid x \in F\right\}$ does not lift to a subgroup of $E$.

We define a basis for $W$, and write down the images under $A_{x}$ :

$$
\begin{aligned}
& w_{1}=e_{1} \wedge e_{2}=\left(f_{3} \wedge f_{4}\right)^{\phi} \mapsto w_{1} ; \\
& w_{2}=e_{1} \wedge e_{3}=\left(f_{2} \wedge f_{4}\right)^{\phi} \mapsto w_{2}+x w_{3}+x w_{4}+x^{2} w_{5} ; \\
& w_{3}=e_{1} \wedge e_{4}=\left(f_{2} \wedge f_{3}\right)^{\phi} \mapsto w_{3}+x w_{5} ; \\
& w_{4}=e_{2} \wedge e_{3}=\left(f_{1} \wedge f_{4}\right)^{\phi} \mapsto w_{4}+x w_{5} ; \\
& w_{5}=e_{2} \wedge e_{4}=\left(f_{1} \wedge f_{3}\right)^{\phi} \mapsto w_{5} ; \\
& w_{6}=e_{3} \wedge e_{4}=\left(f_{1} \wedge f_{2}\right)^{\phi} \mapsto w_{6} .
\end{aligned}
$$

Let $v_{x}=\left(e_{1}+x e_{2}, f_{1}\right)$ and let $u=\left(e_{3}, f_{3}\right)$. Then $v_{x} \perp u$ for all $x \in F$. The voltage along the dart between $u$ and $v_{x}$ is

$$
\left(\left(e_{1}+x e_{2}\right) \wedge e_{3}\right)\left(f_{1} \wedge f_{3}\right)=w_{2} w_{5}+x w_{4} w_{5} .
$$

We choose $\lambda$ as in Lemma 2.4 and take $v=v_{0}$, so $v^{A_{x}}=v_{x}$. For all $x \in F$, we choose a walk $P_{A_{x}}$ as $\left(v_{x}, u, v\right)$. Then $\lambda\left(A_{x}\right)=x w_{4} w_{5}$. Hence

$$
f\left(A_{x}, A_{y}\right)=\lambda\left(A_{x+y}\right)+\lambda\left(A_{x}\right)^{A_{y}}+\lambda\left(A_{y}\right)=x y w_{5}^{2} \in M .
$$

Now suppose that $A$ lifts to a subgroup of $E$, that is, there is a function $c: F \rightarrow M$ such that $\{[x, c(x)] \mid x \in F\}$ with multiplication as in Eq. (4) is a group isomorphic to $A$. Then $[1, c(1)],[\alpha, c(\alpha)]$, and $[\alpha+1, c(\alpha+1)]$ need to have order 2 .

Now $[x, m]^{2}=\left[0, x^{2} w_{5}^{2}+m^{A_{x}}+m\right]$, so $[x, m]$ has order two if and only if $m^{A_{x}}+m=$ $x^{2} w_{5}^{2}$. By elementary linear algebra we find that this is true for $x \neq 0$ if and only if $m \in w_{3}^{2}+S$, where $S=\left\langle w_{1}^{2}, w_{3}^{2}+w_{4}^{2}, w_{5}^{2}, w_{6}^{2}\right\rangle_{\mathbb{F}}$. Note that $S$ is $A$-invariant.

Let $s(x)=w_{3}^{2}+c(x)$. Then $s(x) \in S$. Since $[1, c(1)][\alpha, c(\alpha)]=[\alpha+1, c(\alpha+1)]$, we have $s(\alpha+1)=w_{3}^{2}+\alpha w_{5}^{2}+c(1)^{A_{\alpha}}+c(\alpha)=w_{3}^{2}+\left(\alpha+\alpha^{2}\right) w_{5}^{2}+s(1)+s(\alpha) \notin S$, a contradiction.

Since $A$ does not lift to a subgroup of $E$, neither does $\mathrm{SL}_{4}(\mathbb{F})$. In other words, the extension of $\mathrm{SL}_{4}(\mathbb{F})$ by $M$ is non-split.

\section{References}

1. G. W. Bell, "On the cohomology of the finite special linear groups," J. Algebra 54 (1978), 216-259.

2. N. Biggs, Algebraic Graph Theory, Cambridge, Cambridge University Press, 1974. 
3. K.S. Brown, Cohomology of Groups, New York, Springer-Verlag, 1982.

4. R. Gramlich, On Graphs, Geometries, and Groups of Lie Type, Ph.D. thesis, Technische Universiteit Eindhoven, 2002

5. R.L. Griess, Jr, "Automorphisms of extra special groups and nonvanishing degree 2 cohomology," $P a-$ cific J. Math. 48 (1973), 403-422.

6. J. L. Gross and Th. W. Tucker, Topological Graph Theory, New York, John Wiley \& Sons, 1987.

7. A. Malnič, R. Nedela, and M. Škoviera, "Lifting graph automorphisms by voltage assignments," Europ. J. Combinatorics 21 (2000), 927-947.

8. E. J. Postma, "Covers of graphs related to the general linear group," M.Sc. thesis, Technische Universiteit Eindhoven, 2003.

9. C.-H. Sah, "Cohomology of split group extensions, II," J. Algebra 45 (1977), 17-68. 